Pacific Journal of Mathematics

COHOMOLOGY WITH SUPPORTS 


\title{
COHOMOLOGY WITH SUPPORTS
}

\author{
E. SPANIER
}

In this paper we study cohomology theories on a space $X$ with supports in a family of supports $\Phi$. There is a uniqueness theorem asserting that a homomorphism between two cohomology theories on the space $X$ with the same family of supports $\Phi$ which is an isomorphism for every $A \in \Phi$ is an isomorphism for every closed set $A \subset X$.

1. Introduction. By using cohomology with supports in a given family it is possible to pass from cohomology theories on $X$ to cohomology theories on subsets of $X$ with suitably related families of supports. In particular, compactly supported cohomology theories on a locally compact space $X$ correspond to cohomology theories on the one-point compactification of $X$ which vanish at infinity. Similarly, cohomology theories on a locally paracompact space $X$ with relatively paracompact supports correspond to cohomology theories on the one-point paracompactification of $X$ which vanish at infinity.

We also prove a uniqueness theorem for homomorphisms between additive cohomology theories with paracompact supports on finite dimensional space.

The remainder of the paper is divided into four sections. Section 2 contains the definition of a cohomology theory with supports in a family $\Phi$, a uniqueness theorem for two cohomology theories with the same family of supports, and a characterization of cohomology with supports in suitable families in terms of limit properties.

Section 3 is devoted to the construction of cohomology theories on a space $X$ with supports in a given family $\Phi$ from an $E S$ theory on $X$. The definition of an $E S$ theory on $X$ is given and it is shown that given an $E S$ theory on $X$ and a family $\Phi$ of supports on $X$ there is another $E S$ theory on $X$ with supports in $\Phi$.

In Section 4 the relation between cohomology theories on $X$ and on open subsets of $X$ is studied. The concept of a cohomology theory on $X$ concentrated on a subset $Y \subset X$ (i.e. which vanishes for every closed subset of $X$ contained in $X-Y$ ) is introduced. The main result is a bijection between cohomology theories on $X$ concentrated on an open set $Y$ with supports in $\Phi$ and cohomology theories on $Y$ with supports in a suitable family $\Phi \mid Y$. 
The particular cases of compact and paracompact supports are studied in $\$ 5$. Cohomology theories on a locally compact (locally paracompact) space $X$ with compact (relatively paracompact) supports are shown to correspond to cohomology theories on the one-point compactification (paracompactification) $X^{+}$which are concentrated on $X$. There is also established a uniqueness theorem for addititive cohomology theories with paracompact supports on finite dimensional normal spaces.

2. Supports. We consider cohomology theories on a space $X$ with a given family of closed subsets of $X$ as supports. The uniqueness theorem extends to this case and asserts that if $\varphi: H, \delta \rightarrow H^{\prime}, \delta^{\prime}$ is a homomorphism between cohomology theories on the same space $X$ with the same family $\Phi$ of supports such that for some integer $n, \varphi_{A}: H(A) \rightarrow H^{\prime}(A)$ is an $n$-equivalence for all $A \in \Phi$ then $\varphi_{A}$ is an $n$-equivalence for all closed $A \subset X$.

All topological spaces will be assumed to be Hausdorff spaces. $A$ cohomology theory $[7,8] H, \delta$ on $X$ consists of:

(i) a contravariant functor $H$ from the category $\mathrm{cl}(X)$ of closed subsets of $X$ and inclusion maps to the category of graded abelian groups and homomorphisms of degree 0 such that $H(\varnothing)=0$, and

(ii) a natural transformation $\delta: H(A \cap B) \rightarrow H(A \cup B)$ of degree 1 for every two closed sets $A, B$ in $X$, such that the following are satisfied:

Continuity. For closed $A \subset X$ there is an isomorphism

$$
\rho: \lim _{\rightarrow}\{H(N) \mid N \text { a closed nbhd of } A \text { in } X\} \approx H(A)
$$

where $\rho\{u\}=u \mid A$ for $u \in H(N)$.

$M V$ exactness. For $A, B \subset X$ there is an exact sequence

$$
\begin{aligned}
\ldots & \stackrel{\beta}{\rightarrow} H^{q-1}(A \cap B) \stackrel{\delta}{\rightarrow} H^{q}(A \cup B) \stackrel{\alpha}{\rightarrow} H^{q}(A) \oplus H^{q}(B) \\
& \stackrel{\beta}{\rightarrow} H^{q}(A \cap B) \stackrel{\delta}{\rightarrow} \ldots
\end{aligned}
$$

where $\alpha(u)=(u|A, u| B)$ for $u \in H^{q}(A \cup B)$ and $\beta(u, v)=u \mid A \cap B$ $-v \mid A \cap B$ for $u \in H^{q}(A), v \in H^{q}(B)$.

A cohomology theory $H, \delta$ is said to be non negative if $H^{q}(A)=0$ for $q<0$ and all closed $A \subset X$. A cohomology theory $H, \delta$ is said to be 
additive if for every discrete ${ }^{1}$ family $\left\{A_{j}\right\}$ of closed sets there is an isomorphism

$$
H\left(\bigcup A_{j}\right) \approx \prod H\left(A_{j}\right)
$$

sending $u \in H\left(\bigcup A_{j}\right)$ to $\left\{u \mid A_{j}\right\}$.

A family of supports [1] $\Phi$ on $X$ consists of a collection of closed subsets of $X$ such that

(i) $A \in \Phi, B$ closed in $A \Rightarrow B \in \Phi$.

(ii) $A, B \in \Phi \Rightarrow A \cup B \in \Phi$.

If $\Phi$ also has the property

(iii) $A \in \Phi \Rightarrow$ there is a closed nbhd $N$ of $A$ in $X$ with $N \in \Phi$, we say $\Phi$ is a nbhd family of supports.

EXAmples (2.1). The collection of all closed subsets of $X$ is a family of supports on $X$. In case $X$ is a normal space, it is a nbhd family of supports.

(2.2). The collection of all compact subsets of $X$ is a family of supports on $X$. In case $X$ is locally compact, it is a neighborhood family of supports.

(2.3). The collection of all paracompact subsets of $X$ is a family of supports on $X$. The collection of all closed subsets of $X$ having closed paracompact nbhds in $X$ is a nbhd family of supports on $X$.

(2.4). If $Y \subset X$ and $\Phi$ is a family of supports on $X$, then $\Phi \mid Y=$ $\{A \in \Phi \mid A \subset Y\}$ is a family of supports on $Y$ and on $X$. If $Y$ is open in a normal space $X$ and $\Phi$ is a nbhd family in $X$, then $\Phi \mid Y$ is a nbhd family on $Y$ and on $X$.

If $\Phi$ is a family of supports on $X$ and $H, \delta$ is a cohomology theory on $X$, then $H, \delta$ has supports in $\Phi$ if given $u \in H(A)$ there exist $B$ closed, $C \in \Phi$ with $A=B \cup C$ and $u \mid B=0$.

This definition is a generalization of compactly supported cohomology $[7,8]$. Note that the definition does not involve the natural transformation $\delta$. Obviously every cohomology theory on $X$ has supports in the family of all closed subsets of $X$.

If $H, \delta$ and $H^{\prime}, \delta^{\prime}$ are cohomology theories on the same space $X$, a homomorphism $\varphi$ from $H, \delta$ to $H^{\prime}, \delta^{\prime}$ is a natural transformation from $H$ to $H^{\prime}$ commuting up to sign with $\delta, \delta^{\prime}$.

${ }^{1} \mathrm{~A}$ family $\left\{A_{j}\right\}$ of subsets of a space $X$ is discrete if every point of $X$ has a nbhd meeting at most one member of the family. 
The following is a generalization of [8, Proposition (2.8)] to arbitrary families of supports.

THEOREM (2.5). Let $\varphi: H, \delta \rightarrow H^{\prime}, \delta^{\prime}$ be a homomorphism between two cohomology theories on $X$ with supports in $\Phi$ and suppose $n \in \mathbf{Z}$ is such that $\varphi_{A}: H(A) \rightarrow H^{\prime}(A)$ is an n-equivalence ${ }^{2}$ for every $A \in \Phi$. Then $\varphi_{A}$ is an $n$-equivalence for every closed $A \subset X$.

Proof. The proof parallels that of [8, Proposition (2.8)] and will, therefore, be omitted.

Given a family $\Phi$ of supports on $X$ a set $S \subset X$ is a co- $\Phi$ set if $\overline{X-S} \in \Phi$. The following is a useful criterion for verifying that a contravariant functor on $\operatorname{cl}(X)$ is continuous and has supports $\Phi$.

Proposition (2.6). Assume $H$ is a contravariant functor from $\mathrm{cl}(X)$ to the category of graded abelian groups such that $H(\varnothing)=0, \Phi$ is a family of supports on $X$, and for every closed $A \subset X$ there is an isomorphism

$$
\rho: \lim _{\rightarrow}\{H(N) \mid N \text { a closed co- } \Phi \text { nbhd of } A \text { in } X\} \approx H(A)
$$

where $\rho\{u\}=u \mid A$ for $u \in H(N)$. Then $H$ is continuous and has supports in $\Phi$.

Proof. We first show $H$ is continuous. Let $\rho^{\prime}: \lim _{\rightarrow}\{H(N) \mid N$ a closed neighborhood of $A$ in $x\} \rightarrow H(A)$ be the map of the continuity property. Then (*) implies that $\rho^{\prime}$ is surjective. To show it injective assume $N$ is a closed neighborhood of $A$ in $X$ and $u \in H(N)$ is such that $u \mid A=0$. By (*) there is a closed co- $\Phi$ neighborhood $\bar{N}$ of $N$ in $X$ and $\bar{u} \in H(\bar{N})$ such that $u=\bar{u} \mid N$. Then $\bar{u} \mid A=0$ so, again by $(*)$, there is a closed co- $\Phi$ neighborhood $M$ of $A$ in $\bar{N}$ such that $\bar{u} \mid M=0$. Then $N \cap M$ is a closed neighborhood of $A$ in $N$ and $u|N \cap M=\bar{u}| N \cap M=0$ proving that $\rho^{\prime}$ is injective. Therefore, $H$ is continuous.

To show $H$ has supports in $\Phi$ let $u \in H(A)$. By (*) there is a closed co- $\Phi$ neighborhood $N$ of $A$ in $X$ and $v \in H(N)$ such that $v \mid A=u$. Since $v \mid \varnothing=0$ because $H(\varnothing)=0$, it follows from $(*)$ again that there is a closed co- $\Phi M$ in $N$ such that $v \mid M=0$. Let $B=A \cap M$ and $C$ $=\overline{A-M}$. Then $A=B \cup C$ where $B$ is closed, $C \in \Phi$ and $u \mid B=0$.

\footnotetext{
${ }^{2} \mathrm{~A}$ homomorphism $\varphi: G \rightarrow G^{\prime}$ of degree 0 between graded abelian groups is an n-equivalence if $\varphi: G^{q} \rightarrow G^{q}$ is an isomorphism for all $q<n$ and a monomorphism for $q=n$.
} 
In the case of nbhd families of supports and for cohomology theories $H, \delta$ there is the following converse of Proposition (2.6).

Proposition (2.7). Assume $H, \delta$ is a cohomology theory on $X$ with supports in a nbhd family $\Phi$. Then there is an isomorphism

$\rho: \lim _{\rightarrow}\{H(N) \mid N$ a closed co- $\Phi$ nbhd of $A$ in $X\} \approx H(A)$

where $\rho\{u\}=u \mid A$ for $u \in H(N)$.

Proof. (1) Let $u \in H(A)$ and suppose $M$ is a closed nbhd of $A$ and $v \in H(M)$ are such that $v \mid A=u$ (such $M, v$ exist because $H$ is continuous). Since $H$ has supports in $\Phi, M=B \cup C$ where $B$ is closed, $C \in \Phi$ and $v \mid B=0$. Since $\Phi$ is a nbhd family there is a closed nbhd $C^{\prime}$ of $C$ with $C^{\prime} \in \Phi$. Let $N=M \cup\left(X-\operatorname{int} C^{\prime}\right)$. Then $N$ is a closed nbhd of $A$ in $X$ and

$$
\overline{X-N}=\overline{X-M} \cap \overline{\operatorname{int} C^{\prime}} \subset C^{\prime}
$$

so $N$ is a co- $\Phi$ set. Since $C \cap\left[B \cup\left(X-\operatorname{int} C^{\prime}\right)\right]=C \cap B$, there is an exact sequence

$$
H(N) \stackrel{\alpha}{\rightarrow} H(C) \oplus H\left(B \cup\left(X-\operatorname{int} C^{\prime}\right)\right) \stackrel{\beta}{\rightarrow} H(C \cap B) .
$$

Since $(v \mid C, 0) \in H(C) \oplus H\left(B \cup\left(X-\operatorname{int} C^{\prime}\right)\right)$ is in $\operatorname{ker} \beta$, there is $w \in$ $H(N)$ such that $w|C=v| C$ and $w \mid\left[B \cup\left(X-\operatorname{int} C^{\prime}\right)\right]=0$. Then $w \mid M$ and $v$ have the same restrictions to $C$ and to $B$ so by exactness of

$$
H(C \cap B) \stackrel{\delta}{\rightarrow} H(M) \stackrel{\alpha}{\rightarrow} H(C) \oplus H(B)
$$

there is $w^{\prime} \in H(C \cap B)$ such that $\delta w^{\prime}=v-w \mid M$. There is a commutative diagram

$$
\begin{aligned}
& H\left(C \cap\left[\left(B \cup\left(X \text {-int } C^{\prime}\right)\right)\right]\right)=H(C \cap B) \\
& \delta^{\prime} \downarrow \quad \downarrow \delta \\
& H(N) \stackrel{\rho}{\rightarrow} H(M) .
\end{aligned}
$$

It follows that $v=\delta w^{\prime}+w\left|M=\left(\delta^{\prime} w^{\prime}+w\right)\right| M$. Therefore, $\delta^{\prime} w^{\prime}+w \in$ $H(N)$ is such that

$$
\left(\delta^{\prime} w^{\prime}+w\right)\left|A=\left(\left(\delta^{\prime} w^{\prime}+w\right) \mid M\right)\right| A=v \mid A=u .
$$

This proves that the map $\rho$ in the Proposition is an epimorphism.

(2) To show $\rho$ is a monomorphism assume $u \in H(N)$ where $N$ is a closed co- $\Phi$ nbhd of $A$ in $X$ is such that $u \mid A=0$. Since $H$ has supports 
in $\Phi, N=B \cup C$ with $B$ closed, $C \in \Phi$ and $u \mid B=0$. By continuity of $H$ there is also a closed nbhd $M$ of $A$ in $N$ such that $u \mid M=0$. There is an exact sequence

$$
H(M \cap B) \stackrel{\delta}{\rightarrow} H(M \cup B) \stackrel{\alpha}{\rightarrow} H(M) \oplus H(B)
$$

and $u \mid(M \cup B)$ is in $\operatorname{ker} \alpha$ so there is $v \in H(M \cap B)$ with $\delta v=$ $u \mid(M \cup B)$. By (1) above there are a closed co- $\Phi$ nbhd $L$ of $M \cap B$ in $N$ and $w \in H(L)$ such that $w \mid M \cap B=v$. Clearly $L=(M \cup L) \cap(B \cup L)$ and there is a commutative diagram

$$
\begin{array}{ccccc}
H(L) & \stackrel{\delta^{\prime}}{\rightarrow} & H(M \cup L \cup B) & \stackrel{\alpha^{\prime}}{\rightarrow} & H(M \cup L) \oplus H(B \cup L) \\
\rho^{\prime} \downarrow & & \rho \downarrow & & \downarrow \rho^{\prime \prime} \\
H(M \cap B) & \stackrel{\delta}{\rightarrow} & H(M \cup B) & \stackrel{\alpha}{\rightarrow} & H(M) \oplus H(B)
\end{array}
$$

Since $M \cup B, M \cup L$ are closed co- $\Phi$ nbhds of $A$ in $N$ (because $\overline{X-M \cup B}=\overline{X-M} \cap \overline{X-B} \subset \overline{X-B}=\overline{X-N} \cup \overline{N-B} \in \Phi$ and $\overline{X-M \cup L} \subset \overline{X-L} \in \Phi)$, it follows that $D=(M \cup B) \cap(M \cup L)$ is a closed co- $\Phi$ nbhd of $A$ in $N$. Clearly

$$
\begin{aligned}
u \mid D & =(u \mid(M \cup B))|D=(\delta v)| D=\left(\delta \rho^{\prime} w\right)\left|D=\left(\rho \delta^{\prime} w\right)\right| D \\
& =\left(\left(\delta^{\prime} w\right) \mid(M \cup L)\right)|D=0| D=0
\end{aligned}
$$

proving that $\rho$ is a monomorphism.

3. Existence of cohomology with given supports. In this Section we show how to obtain cohomology theories on a normal space $X$ with supports in a given nbhd family of supports $\Phi$ from an $E S$ theory on $X$. We begin by recalling the definition of an ES theory on $X$ and some of its properties. See [8] for more details.

As ES theory $H, \delta^{*}$ on $X$ consists of:

(i) a contravariant functor $H$ from $\operatorname{cl}(X)^{2}$ (the category of closed pairs in $X$ and inclusion maps between them) to the category of graded abelian groups, and

(ii) a natural transformation

$$
\delta^{*}: H(B, \varnothing) \rightarrow H(A, B)
$$

of degree 1 for every closed pair $(A, B)$ in $X$, such that the following are satisfied:

Continuity. For every closed $A$ in $X$ there is an isomorphism

$$
\rho: \lim _{\rightarrow}\{H(N, \varnothing) \mid N \text { a closed nbhd of } A \text { in } X\} \approx H(A, \varnothing)
$$

where $\rho\{u\}=u \mid(A, \varnothing)$ for $u \in H(N, \varnothing)$. 
Exactness. For every closed pair $(A, B)$ in $X$ the following sequence is exact

$$
\ldots \stackrel{\delta^{*}}{\rightarrow} H^{q}(A, B) \stackrel{H(j)}{\rightarrow} H^{q}(A, \varnothing) \stackrel{H(i)}{\rightarrow} H^{q}(B, \varnothing) \stackrel{\delta^{*}}{\rightarrow} H^{q+1}(A, B) \rightarrow \ldots
$$

where $i:(B, \varnothing) \subset(A, \varnothing)$ and $j:(A, \varnothing) \subset(A, B)$.

Excision. For closed sets $A, B$ in $X$ there is an isomorphism

$$
\rho: H(A \cup B, B) \approx H(A, A \cap B)
$$

It is standard [2] that if $(A, B, C)$ is a closed triple in $X$ there is a corresponding exact sequence of the triple and that there is a cohomology theory $H^{\prime}, \delta^{\prime}$ on $X$ with $H^{\prime}(A)=H(A, \varnothing)$ for $A \in \operatorname{cl}(X)$. An $E S$ theory has supports in a family $\Phi$ if the corresponding cohomology theory has supports in $\Phi$.

The following will be useful in constructing ES theories with supports in $\Phi$.

LEMMA (3.1). Let $\Phi$ be a nbhd family of supports on a normal space $X$. If $A$ is closed and $N$ is a co- $\Phi$ nbhd of $A$ in $X$, there is a closed co- $\Phi$ nbhd of $A$ contained in the interior of $N$.

Proof. By hypothesis $A$ is disjoint from $\overline{X-N} \in \Phi$. Let $M \in \Phi$ be a nbhd of $\overline{X-N}$. Since $A$ and $\overline{X-N}$ are disjoint closed sets in $X$ there exist disjoint closed nbhds $A^{\prime}$ of $A$ and $B^{\prime}$ of $\overline{X-N}$. Then $B^{\prime} \cap M \in \Phi$ is a nbhd of $\overline{X-N}$ disjoint from $A^{\prime}$. Therefore, $N^{\prime}=\overline{X-B^{\prime} \cap M}$ is a closed nbhd of $A$ contained in $X-\overline{X-N}=$ interior of $N$ and $\overline{X-N^{\prime}} \subset$ $B^{\prime} \cap M \in \Phi$ so $N^{\prime}$ is a co- $\Phi$ nbhd of $A$ contained in the interior of $N$.

THEOREM (3.2). Let $H, \delta^{*}$ be an ES theory on a normal space $X$ and let $\Phi$ be a nbhd family of supports on $X$. Then there is an ES theory $H_{\Phi}, \delta_{\Phi}^{*}$ on $X$ with supports in $\Phi$ where

$H_{\Phi}(A, B)=\lim _{\rightarrow}\{H(M, N) \mid(M, N)$ a closed co- $\Phi$ nbhd of $(A, B)$ in $X\}$.

Proof. Note that the intersection of two closed co- $\Phi$ nbhds of $(A, B)$ is a closed co- $\Phi$ nbhd of $(A, B)$ so the collection of closed co- $\Phi$ nbhds of $(A, B)$ is directed downward by inclusion and we can define $H_{\Phi}(A, B)=\lim _{\rightarrow}\{H(M, N) \mid(M, N)$ a closed co- $\Phi$ nbhd of $(A, B)$ in $X\}$, and $H_{\Phi}$ is a contravariant functor on $\operatorname{cl}(X)^{2}$. 
Consider closed triples $(M, N, P)$ of co- $\Phi$ sets such that $(M, N)$ is a nbhd of $(A, B)$. As $(M, N, P)$ vary over the collection of all such triples (which is directed downward by inclusion) note that:

(1) $(M, N)$ varies over all closed co- $\Phi$ nbhds of $(A, B)$ in $X$ (to such $(M, N)$ there is the triple $(M, N, N))$,

(2) $(M, P)$ varies over all closed co- $\Phi$ nbhds of $(A, \varnothing)$ (to such $(M, P)$ there is the triple $(M, M, P))$, and

(3) $(N, P)$ varies over all closed co- $\Phi$ nbhds of $(B, \varnothing)$ (to such $(N, P)$ there is the triple $(X, N, P))$.

Corresponding to such a triple $(M, N, P)$ there is an exact sequence

$$
\ldots \rightarrow H^{q}(M, N) \rightarrow H^{q}(M, P) \rightarrow H^{q}(N, P) \stackrel{\delta^{*}}{\rightarrow} H^{q+1}(M, N) \rightarrow \ldots
$$

Taking the direct limit of these exact sequences over all such triples $(M, N, P)$ and using (1), (2), (3) we obtain an exact sequence

$$
\ldots \rightarrow H_{\Phi}^{q}(A, B) \rightarrow H_{\Phi}^{q}(A, \varnothing) \rightarrow H_{\Phi}^{q}(B, \varnothing) \stackrel{\delta_{\Phi}^{*}}{\rightarrow} H_{\Phi}^{q+1}(A, B) \rightarrow \ldots
$$

This defines the natural transformation $\delta_{\Phi}^{*}$ of degree 1 such that $H_{\Phi}, \delta_{\Phi}^{*}$ satisfy exactness.

We verify excision. Given closed sets $A, B$ in $X$ let $(M, N)$ be a closed co- $\Phi$ nbhd of $(A, A \cap B)$ in $X$ and $\left(M^{\prime}, N^{\prime}\right)$ a closed co- $\Phi$ nbhd of $(A \cup B, B)$ in $X$. Then $A$-int $N \cap N^{\prime}$ and $B$-int $N \cap N^{\prime}$ are disjoint closed subsets of $M^{\prime}$ so there exist disjoint closed nbhds $E$ of $A$-int $N \cap N^{\prime}$ and $F$ of $B$-int $N \cap N^{\prime}$ in $M^{\prime}$. Then $M^{\prime \prime}=\left[E \cup\left(N \cap N^{\prime}\right)\right] \cap M$ is a closed co- $\Phi$ nbhd of $A$ contained in $M^{\prime} \cap M$ and $N^{\prime \prime}=(F \cup N) \cap N^{\prime}$ is a closed co- $\Phi$ nbhd of $B$ contained in $N^{\prime}$ such that $M^{\prime \prime} \cup N^{\prime \prime} \subset M^{\prime}$ and $M^{\prime \prime} \cap N^{\prime \prime}=N \cap N^{\prime} \cap M \subset N$. Thus, $\left(M^{\prime \prime}, N^{\prime \prime} \cap M^{\prime \prime}\right)$ is a closed co- $\Phi$ nbhd of $(A, A \cap B)$ contained in $(M, N)$ and $\left(M^{\prime \prime} \cup N^{\prime \prime}, N^{\prime \prime}\right)$ is a closed co- $\Phi$ nbhd of $(A \cup B, B)$ contained in $\left(M^{\prime}, N^{\prime}\right)$. Since $H$ satisfies excision,

$$
H\left(M^{\prime \prime} \cup N^{\prime \prime}, N^{\prime \prime}\right) \approx H\left(M^{\prime \prime}, M^{\prime \prime} \cap N^{\prime \prime}\right) .
$$

Since this isomorphism is valid for a cofinal system of closed co- $\Phi$ nbhds of $(A \cup B, B)$ and of $(A, A \cup B)$ on taking direct limits we obtain an isomorphism

$$
H_{\Phi}(A \cup B, B) \approx H_{\Phi}(A, A \cap B) .
$$

To complete the proof we show that $H_{\Phi}$ is continuous and has supports in $\Phi$. Since $H_{\Phi}(\varnothing, \varnothing)=0$, in view of Proposition (2.6) it suffices to verify that the homomorphism

$$
\rho: \lim _{\rightarrow}\left\{H_{\Phi}(M, \varnothing) \mid M \text { a closed co- } \Phi \text { nbhd of } A\right\} \rightarrow H_{\Phi}(A, \varnothing)
$$

is an isomorphism. 
Let $u \in H_{\Phi}(A, \varnothing)$. By definition of $H_{\Phi}$ there is a closed co- $\Phi$ nbhd $(M, N)$ of $(A, \varnothing)$ and $v \in H(M, N)$ such that $u=\{v\}_{(A, \varnothing)}$. By Lemma (3.1) there is a closed co- $\Phi$ nbhd $M^{\prime}$ of $A$ contained in int $M$. Then $v$ determines $\{v\}_{\left(M^{\prime}, \varnothing\right)} \in H_{\Phi}\left(M^{\prime}, \varnothing\right)$ such that

$$
\{v\}_{\left(M^{\prime}, \varnothing\right)} \mid(A, \varnothing)=\{v\}_{(A, \varnothing)}=u
$$

proving that $\rho$ is an epimorphism.

To show that $\rho$ is a monomorphism let $u \in H_{\Phi}(M, \varnothing)$ be such that $u \mid(A, \varnothing)=0$ where $M$ is a closed co- $\Phi$ nbhd of $A$. By definition of $H_{\Phi}$ there is a closed co- $\Phi$ nbhd $\left(M^{\prime}, N^{\prime}\right)$ of $(M, \varnothing)$ and $v \in H\left(M^{\prime}, N^{\prime}\right)$ such that $u=\{v\}_{(M, \varnothing)}$. Since $0=u \mid(A, \varnothing)=\{v\}_{(A, \varnothing)}$ there is a closed co- $\Phi$ nbhd $\left(M^{\prime \prime}, N^{\prime \prime}\right)$ of $(A, \varnothing)$ contained in $\left(M^{\prime}, N^{\prime}\right)$ such that $v \mid\left(M^{\prime \prime}, N^{\prime \prime}\right)=$ 0 . Since $M \cap M^{\prime \prime}$ is a closed co- $\Phi$ nbhd of $A$ it follows from Lemma (3.1) that there is a closed co- $\Phi$ nbhd $P$ of $A$ contained in $\operatorname{int}\left(M \cap M^{\prime \prime}\right)$. Then $\left(M \cap M^{\prime \prime}, M \cap N^{\prime \prime}\right)$ is a closed co- $\Phi$ nbhd of $(P, \varnothing)$ and

$$
u\left|(P, \varnothing)=\{v\}_{(M, \varnothing)}\right|(P, \varnothing)=\left\{v \mid\left(M \cap M^{\prime \prime}, M \cap N^{\prime \prime}\right)\right\}_{(P, \varnothing)}=0
$$

showing that $\rho$ is a monomorphism.

The following is an interesting alternate description of the functor $H_{\Phi}$ defined in Theorem (3.2).

Proposition (3.3). Let $H, \delta^{*}$ be an ES theory on a normal space $X$ and $\Phi$ a nbhd family of supports on $X$. Let $H_{\Phi}$ be the contravariant functor on $\operatorname{cl}(X)^{2}$ defined in Theorem (3.2). For any $(A, B) \in \operatorname{cl}(X)^{2}$ there is an isomorphism

$$
\rho^{\prime}: H_{\Phi}(A, B) \approx \lim _{\overrightarrow{B^{\prime}}}\left\{H\left(A, B^{\prime}\right) \mid B^{\prime} \text { closed }, B \subset B^{\prime} \subset A \text { and } \overline{A-B^{\prime}} \in \Phi\right\}
$$

where $\rho^{\prime}\{v\}_{(A, B)}=\{v \mid(A, N \cap A)\}^{\prime}$ for $v \in H(M, N),(M, N)$ a closed co- $\Phi$ nbhd of $(A, B)$.

Proof. In the above and in the proof we use \{\} to denote elements of $H_{\Phi}(A, B)$ and \{\}$^{\prime}$ to denote elements of the direct limit which is the codomain of $\rho^{\prime}$. It is clear that $\rho^{\prime}$ as defined above is a homomorphism.

We show $\rho^{\prime}$ is an epimorphism. Let $\{v\}_{(A, B)}^{\prime} \in \lim _{B^{\prime}}\left\{H\left(A, B^{\prime}\right) \mid B^{\prime}\right.$

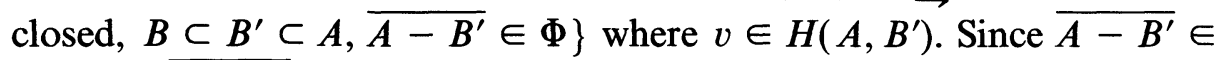
$\Phi, X-\left(\overline{A-B^{\prime}}\right)$ is a co- $\Phi$ nbhd of $\varnothing$ in $X$. By Lemma (3.1) there is a closed co- $\Phi$ nbhd $N$ of $\varnothing$ contained in $\operatorname{int}\left(X-\left(\overline{A-B^{\prime}}\right)\right)=X-$ $\left(\overline{A-B^{\prime}}\right)$. Then $A$ and $B^{\prime} \cup N$ are closed sets such that

$$
A \cap\left(B^{\prime} \cup N\right)=\left[\overline{A-B^{\prime}} \cup B^{\prime}\right] \cap\left[B^{\prime} \cup N\right]=B^{\prime} \text {. }
$$


Therefore, there is an excision isomorphism

$$
H\left(A \cup\left(B^{\prime} \cup N\right), B^{\prime} \cup N\right) \approx H\left(A, B^{\prime}\right) .
$$

Let $v^{\prime} \in H\left(A \cup N, B^{\prime} \cup N\right)$ be such that $v^{\prime} \mid\left(A, B^{\prime}\right)=v$. By continuity of $H$ there is a closed nbhd $\left(M, M^{\prime}\right)$ of $\left(A \cup N, B^{\prime} \cup N\right)$ and $v^{\prime \prime} \in H\left(M, M^{\prime}\right)$ such that $v^{\prime \prime} \mid\left(A \cup N, B^{\prime} \cup N\right)=v^{\prime}$. Then $\left(M, M^{\prime}\right)$ is a closed co- $\Phi$ nbhd of $(A, B)$ so $\left\{v^{\prime \prime}\right\}_{(A, B)} \in H_{\Phi}(A, B)$ and $\rho^{\prime}\left\{v^{\prime \prime}\right\}_{(A, B)}=$ $\left\{v^{\prime \prime} \mid\left(A, A \cap M^{\prime}\right)\right\}_{(A, B)}^{\prime}=\left\{v^{\prime \prime} \mid\left(A, B^{\prime}\right)\right\}_{(A, B)}^{\prime}=\{v\}_{(A, B)}^{\prime}$. So $\rho^{\prime}$ is an epimorphism.

To show $\rho^{\prime}$ is a monomorphism let $u \in H_{\Phi}(A, B)$ be such that $\rho^{\prime}(u)=0$ and let $(M, N)$ be a closed co- $\Phi$ nbhd of $(A, B)$ and $v \in$ $H(M, N)$ be such that $u=\{v\}_{(A, B)}$. Then

$$
0=\rho^{\prime}(u)=\{v \mid(A, A \cap N)\}_{(A, B)}^{\prime}
$$

so there is closed $B^{\prime}, B \subset B^{\prime} \subset A \cap N, \overline{A-B^{\prime}} \in \Phi$ such that $v \mid\left(A, B^{\prime}\right)$ $=0$. Then $N \cap\left[X-\overline{A-B^{\prime}}\right]$ is a co- $\Phi$ nbhd of $\varnothing$ in $X$. By Lemma (3.1) there is a closed co- $\Phi$ nbhd $N^{\prime}$ of $\varnothing$ contained in

$$
\operatorname{int}\left(N \cap\left[X-\overline{A-B^{\prime}}\right]\right)=(\operatorname{int} N) \cap\left[X-\overline{A-B^{\prime}}\right] .
$$

Then $A \cup\left(B^{\prime} \cup N^{\prime}\right)=A \cup N^{\prime}$ and $A \cap\left(B^{\prime} \cup N^{\prime}\right)=B^{\prime}$ so there is an excision isomorphism

$$
H\left(A \cup N^{\prime}, B^{\prime} \cup N^{\prime}\right) \approx H\left(A, B^{\prime}\right) .
$$

Since $v \mid\left(A, B^{\prime}\right)=0$, it follows that $v \mid\left(A \cup N^{\prime}, B^{\prime} \cup N^{\prime}\right)=0$. By continuity of $H$, there is a closed nbhd $\left(M^{\prime \prime}, N^{\prime \prime}\right)$ of $\left(A \cup N^{\prime}, B^{\prime} \cup N^{\prime}\right)$ in $(M, N)$ such that $v \mid\left(M^{\prime \prime}, N^{\prime \prime}\right)=0$. Then $\left(M^{\prime \prime}, N^{\prime \prime}\right)$ is a closed co- $\Phi$ nbhd of $(A, B)$ contained in $(M, N)$ and

$$
u=\{v\}_{(A, B)}=\left\{v \mid\left(M^{\prime \prime}, N^{\prime \prime}\right)\right\}_{(A, B)}=0
$$

proving that $\rho^{\prime}$ is a monomorphism.

4. Cohomology of open subsets. We consider relations between cohomology theories on a space $X$ and cohomology theories on subsets $Y$ of $X$.

A cohomology theory $H, \delta$ on a space $X$ is said to be concentrated on a subset $Y \subset X$ if $H(A)=0$ for all closed $A \subset X-Y$. An $E S$ theory is said to be concentrated on $Y$ if the corresponding cohomology theory is concentrated on $Y$.

ExAmple (4.1). Let $Y$ be a closed subset of a normal space $X$ and let $H, \delta$ be a cohomology theory on $X$. The restriction of $H, \delta$ to $Y$ is a cohomology theory $\bar{H}, \bar{\delta}$ on $Y$ and the direct image of $\bar{H}, \bar{\delta}$ under the 
closed continuous map $i: Y \subset X$ is a cohomology theory $H^{\prime}, \delta^{\prime}$ with $H^{\prime}(A)=H(A \cap Y)$. Clearly $H^{\prime}$ is concentrated on $Y$.

The following shows how to obtain cohomology theories concentrated on an open subset $Y \subset X$ given an $E S$ theory on $X$.

Proposition (4.2). Let $H, \delta^{*}$ be an ES theory on $X$ and let $Y$ be an open subset of $X$. There is an ES theory $H^{\prime}, \delta^{\prime}$ concentrated on $Y$ with $H^{\prime}(A, B)=H(A \cup(X-Y), B \cup(X-Y))$ for closed $(A, B)$ in $X$.

Proof. $H^{\prime}$ as defined in the statement of the Proposition is clearly a contravariant functor on $\mathrm{cl}(X)^{2}$. The exact cohomology sequence of the triple $(A \cup(X-Y), B \cup(X-Y), X-Y)$ in $H, \delta^{*}$ becomes the exact cohomology sequence of the pair $(A, B)$ in $H^{\prime}, \delta^{\prime}$ (this defines the natural transformation

$$
\delta^{\prime}: H^{\prime}(B, \varnothing) \rightarrow H^{\prime}(A, B)
$$

of degree 1 such that $H^{\prime}, \delta^{\prime}$ satisfy exactness). Excision for $H^{\prime}, \delta^{\prime}$ follows from excision for $H, \delta^{*}$. To verify continuity for $H^{\prime}, \delta^{\prime}$ note that

$$
H^{\prime}(A, \varnothing)=H(A \cup(X-Y), X-Y) \approx H(A, A \cap(X-Y)) \text {. }
$$

As $N$ varies over closed nbhds of $A$ in $X, N \cap(X-Y)$ varies over closed nbhds of $A \cap(X-Y)$ in $X-Y$. It follows from continuity of $H$ that

$$
\begin{aligned}
\rho: & \lim _{\rightarrow}\{H(N, N \cap(X-Y)) \mid N \text { a closed nbhd of } A \text { in } X\} \\
& \approx H(A, A \cap(X-Y)) .
\end{aligned}
$$

This implies that

$$
\rho: \lim _{\rightarrow}\left\{H^{\prime}(N, \varnothing) \mid N \text { a closed nbhd of } A \text { in } X\right\} \approx H^{\prime}(A, \varnothing)
$$

and so $H^{\prime}$ satisfies continuity.

Thus, $H^{\prime}, \delta^{\prime}$ is an ES theory on $X$. It is concentrated on $Y$ for if $A \subset X-Y$ then

$$
H^{\prime}(A, \varnothing)=H(A \cup(X-Y), X-Y)=H(X-Y, X-Y)=0 .
$$

LEMMA (4.3). If $H, \delta$ is a cohomology theory on $X$ concentrated on an open set $Y \subset X$, then for every closed $A \subset X$, there is an isomorphism

$$
\rho^{\prime}: H(A \cup(X-Y)) \approx H(A) .
$$


Proof. This is immediate from exactness of

$$
0=H(A \cap(X-Y)) \stackrel{\delta}{\rightarrow} H(A \cup(X-Y)) \stackrel{\alpha}{\rightarrow} H(A) \oplus H(X-Y) \rightarrow 0
$$

and the fact that $H(X-Y)=0$.

The following relates cohomology concentrated on an open subset and cohomology having supports in a nbhd family.

Proposition (4.4). Let $H, \delta$ be a cohomology theory on $X, \Phi$ be a nbhd family of supports on $X$, and $Y$ an open subset of $X$. Then $H, \delta$ has supports in $\Phi$ and is concentrated on $Y$ if and only if $H, \delta$ has supports in $\Phi \mid Y$.

Proof. If $\mathrm{H}, \delta$ has supports in $\Phi \mid \mathrm{Y}$, it clearly has supports in the larger family $\Phi$. We show it is concentrated on $Y$. Assume $A \subset X-Y$ and $u \in H(A)$. Since $H$ has supports in $\Phi \mid Y, A=B \cup C$ where $B$ is closed, $C \in \Phi \mid Y$ and $u \mid B=0$. Since $C \subset A \cap Y=\varnothing, B=A$ so $u=$ $u \mid B=0$. Therefore, $H(A)=0$ so $H$ is concentrated on $Y$.

Conversely, assume $H$ has supports in $\Phi$ and is concentrated on $Y$. Let $u \in H(A)$ where $A$ is closed in $X$. By Lemma (4.3) there is $u^{\prime} \in$ $H((A \cup(X-Y)))$ such that $u^{\prime} \mid A=u$. Since $\Phi$ is a nbhd family, it follows from Proposition (2.7) that there is a closed co- $\Phi$ nbhd $N$ of $A \cup(X-Y)$ in $X$ and an element $v \in H(N)$ such that $v \mid[A \cup(X-Y)]=u^{\prime}$. Since $H$ is concentrated on $Y, v \mid(X-Y)=$ 0 . Again, by Proposition (2.7), there is a closed co- $\Phi$ nbhd $M$ of $X-Y$ contained in $N$ such that $v \mid M=0$. Since $M$ is a nbhd of $X-Y$, $\overline{X-M} \subset Y$ so $\overline{X-M} \in \Phi \mid Y$. Then $A=(A \cap M) \cup(\overline{A-M})$ where $A \cap B$ is closed,

$$
\overline{A-M}=A \cap \overline{X-M} \in \Phi \mid Y
$$

and

$$
u|(A \cap M)=(v \mid A)|(A \cap M)=(v \mid M) \mid(A \cap M)=0 .
$$

Hence, $H$ has supports in $\Phi \mid Y$.

The next result asserts, for $Y$ open in a normal space $X$ and $\Phi$ a nbhd family of supports on $X$, that cohomology theories on $X$ with supports in $\Phi \mid Y$ are essentially the same as cohomology theories on $Y$ with supports in $\Phi \mid Y$.

THEOREM (4.5). Given $Y$ open in a normal space $X$ and given $\Phi$ a nbhd family of supports on $X$, there is a bijection between cohomology theories $H$, $\delta$ on $X$ with supports in $\Phi \mid Y$ and cohomology theories $H^{\prime}, \delta^{\prime}$ on $Y$ with supports $\Phi \mid Y$ such that, for $A$ closed in $Y, H^{\prime}(A)=H(A \cup(X-Y))$. 
Proof. Given $H, \delta$ on $X$ for $A$ closed in $Y$ define $H^{\prime}(A)=$ $H(A \cup(X-Y))$, and for closed $A, B$ in $Y$ define $\delta^{\prime}: H^{\prime}(A \cap B) \rightarrow$ $H^{\prime}(A \cup B)$ to equal

$$
\begin{aligned}
\delta: H([A \cup(X-Y)] \cap[B \cup(X-Y)]) \\
\rightarrow H([A \cup(X-Y)] \cup[B \cup(X-Y)]) .
\end{aligned}
$$

Then $\delta^{\prime}$ is a natural transformation of degree 1 such that $H^{\prime}, \delta^{\prime}$ satisfy $M V$ exactness. By Proposition (4.4), $H$ is concentrated on $Y$ so that

$$
H^{\prime}(\varnothing)=H(X-Y)=0 .
$$

By Proposition (2.7) there is an isomorphism

$\rho: \lim _{\rightarrow}\{H(N) \mid N$ a closed co- $\Phi \mid Y$ nbhd of $A \cup(X-Y)$ in $X\}$

$$
\approx H(A \cup(X-Y)) \text {. }
$$

It is clear that $N$ is a closed co- $\Phi \mid Y$ nbhd of $A \cup(X-Y)$ if and only if $N=(N \cap Y) \cup(X-Y)$ where $M=N \cap Y$ is a closed co- $\Phi \mid Y$ nbhd of $A$ in $Y$. Therefore, there is an isomorphism

$\rho: \lim _{\rightarrow}\left\{H^{\prime}(M) \mid M\right.$ a closed co- $\Phi \mid Y$ nbhd of $A$ in $\left.Y\right\} \approx H^{\prime}(A)$.

By Proposition (2.6) $H^{\prime}$ is continuous on $Y$ and has supports in $\Phi \mid Y$. Therefore, $H^{\prime}, \delta^{\prime}$ is a cohomology theory on $Y$ with supports in $\Phi \mid Y$.

Conversely, let $H^{\prime}, \delta^{\prime}$ be a cohomology theory on $Y$ with supports in $\Phi \mid Y$. Define a contravariant functor $H$ on $\operatorname{cl}(X)$ by $H(A)=H^{\prime}(A \cap Y)$ for $A$ closed in $X$. Also, for $A, B$ closed in $X$ define $\delta: H(A \cap B) \rightarrow$ $H(A \cup B)$ to equal

$$
\delta^{\prime}: H^{\prime}((A \cap Y) \cap(B \cap Y)) \rightarrow H^{\prime}((A \cap Y) \cup(B \cap Y)) .
$$

Then $\delta$ is a natural transformation of degree 1 such that $H, \delta$ satisfy $M V$ exactness. By definition, if $A \subset X-Y, H(A)=H^{\prime}(A \cap Y)=$ $H^{\prime}(\varnothing)=0$.

By Proposition (2.7) there is an isomorphism

$$
\begin{aligned}
\rho: & \lim _{\rightarrow}\left\{H^{\prime}(M) \mid M \text { a closed co- } \Phi \mid Y \text { nbhd of } A \cap Y \text { in } Y\right\} \\
& \approx H^{\prime}(A \cap Y) .
\end{aligned}
$$

It is clear that $M$ is a closed co- $\Phi \mid Y$ nbhd of $A \cap Y$ in $Y$ if and only if $N=M \cup(X-Y)$ is a closed co- $\Phi \mid Y$ nbhd of $A$ in $X$. Therefore, there is an isomorphism

$\rho: \lim _{\rightarrow}\{H(N) \mid N$ a closed co- $\Phi \mid Y$ nbhd of $A$ in $X\} \approx H(A)$. 
By Proposition (2.6), $H$ is continuous and has supports in $\Phi \mid Y$. Therefore, $H, \delta$ is a cohomology theory on $X$ with supports in $\Phi \mid Y$.

Given $H, \delta$ on $X$ let $H^{\prime}, \delta^{\prime}$ be the corresponding cohomology theory on $Y$ and $\bar{H}, \bar{\delta}$ the cohomology theory on $X$ corresponding to $H^{\prime}, \delta^{\prime}$. Then for closed $A \subset X$,

$$
\bar{H}(A)=H^{\prime}(A \cap Y)=H((A \cap Y) \cup(X-Y))=H(A \cup(X-Y)) .
$$

Since $H$ is concentrated on $Y$ by Proposition (4.4), Lemma (4.3) implies that $H(A \cup(X-Y)) \approx H(A)$. Thus, $\bar{H} \approx H$ and similarly $\bar{\delta}$ corresponds to $\delta$.

Given $H^{\prime}, \delta^{\prime}$ on $Y$ let $H, \delta$ be the corresponding cohomology theory on $X$ and $H^{\prime \prime}, \delta^{\prime \prime}$ the cohomology theory on $Y$ defined by $H, \delta$. Then, for $A$ closed in $Y$,

$$
H^{\prime \prime}(A)=H(A \cup(X-Y))=H^{\prime}([A \cup(X-Y)] \cap Y)=H^{\prime}(A)
$$

and similarly $\delta^{\prime \prime}$ corresponds to $\delta$. Thus, the passage from $H, \delta$ on $X$ to $H^{\prime}, \delta^{\prime}$ on $Y$ is a bijection of cohomology theories with supports in $\Phi \mid Y$.

Combining the last two results we obtain:

COROllary (4.6). If $Y$ is an open subset of a normal space $X$ and $\Phi$ is a nbhd family on $X$ there is a bijection between cohomology theories $H, \delta$ on $X$ with supports in $\Phi$ concentrated on $Y$ and cohomology theories $H^{\prime}, \delta^{\prime}$ on $Y$ with supports in $\Phi \mid Y$.

5. Compact and paracompact supports. We consider the special cases of the nbhd family of compact supports in a locally compact space and the nbhd family of relatively paracompact supports in a locally paracompact space. We also consider the uniqueness theorem for compactly supported and paracompactly supported cohomology.

Given a topological space $X$ let $\Phi_{c}$ be the nbhd family of all closed subsets of $X$ having a compact nbhd in $X$ and let $X_{l c}=\bigcup\left\{A \in \Phi_{c}\right\}$. Then $X_{l c}$ is the union of all locally compact open subsets of $X$ so is the largest open subset of $X$ which is locally compact. Clearly $\Phi_{c} \mid X_{l c}=\Phi_{c}$ and $\Phi_{c}$ is exactly the family of all compact subsets in $X_{l c}$. It follows from Theorem (4.5) that cohomology theories on $X$ with supports in $\Phi_{c}$ correspond bijectively to compactly supported cohomology theories on the locally compact space $X_{l c}$. Thus, the study of cohomology theories with supports in $\Phi_{c}$ is reduced to the study of compactly supported cohomology theories on locally compact spaces. 
Our next result implies that the compactly supported cohomology theories on a locally compact space correspond to cohomology theories on its one-point compactification which are concentrated on the space.

THEOREM (5.1). Let $X$ be an open subset of a compact space $Z$. There is a bijection between compactly supported cohomology theories on $X$ and cohomology theories on $Z$ which are concentrated on $X$.

Proof. If $\Phi$ is the nbhd family of all closed (or, equivalently, compact) subsets of $Z$, then every cohomology theory on $Z$ has supports in $\Phi$. Clearly $\Phi \mid X=\Phi_{c}$ the nbhd family of all compact subsets of $X$. The theorem follows from Corollary (4.6).

We consider similar definitions for the paracompact rather than the compact case. Given a space $X$ let $\Phi_{p}$ be the family of all closed subsets of $X$ having a paracompact nbhd in $X$. Clearly $X_{p}$ is a nbhd family of supports and if $X_{l p}=\bigcup\left\{A \in \Phi_{p}\right\}$, then $X_{l p}$ is the largest open subset of $X$ which is locally paracompact. Obviously $\Phi_{p} \mid X_{l p}=\Phi_{p}$, but in this case $\Phi_{p}$ is not the family of all paracompact subsets of $X_{l p}$ but is the family of all closed subsets of $X_{l p}$ having paracompact nbhds in $X_{l p}$. It can be shown that this family is identical to the family of all relatively paracompact subsets of $X_{l p}$ (a subset $A$ of a space $X$ is relatively paracompact if given a collection $\mathscr{U}$ of open subsets of $X$ covering $A$ there is a collection $\mathscr{V}$ of open subsets of $X$ covering $A$ which refines $\mathscr{U}$ and is locally finite in $X$ ). In case $X$ is paracompact the family $\Phi_{p}=$ the family of all closed sets.

It follows from Theorem (4.5) that cohomology theories on $X$ with supports in $\Phi_{p}$ correspond bijectively to cohomology theories on the locally paracompact space $X_{l p}$ with relatively paracompact supports. The following implies that cohomology theories with relatively paracompact supports on a locally paracompact space correspond to cohomology theories on its one-point paracompactification which are concentrated on the space.

THeOREM (5.2). Let $X$ be an open subset of a paracompact space $Z$. There is a bijection between cohomology theories on $X$ with relatively paracompact supports and cohomology theories on $Z$ which are concentrated on $X$.

Proof. Analogously to Theorem (5.1) this follows from Corollary (4.6). 
Let $\varphi: H, \delta \rightarrow H^{\prime}, \delta^{\prime}$ be a homomorphism between compactly supported cohomology theories on the same space $X$ such that for some $n \in \mathbf{Z}, \varphi_{x}: H(x) \rightarrow H^{\prime}(x)$ is an $n$-equivalence for all $x \in X$. We would like to deduce that $\varphi_{A}: H(A) \rightarrow H^{\prime}(A)$ is an $n$-equivalence for all closed $A \subset X$. In case $H, H^{\prime}$ are nonnegative if follows from [7, Theorem 3.1] and in case $X$ is a finite dimensional separable metric case it follows from [8, Corollary (4.3)].

Now suppose $\varphi: H, \delta \rightarrow H^{\prime}, \delta^{\prime}$ is a homomorphism between additive paracompactly supported cohomology theories on $X$ such that for some $n \in \mathbf{Z}, \varphi_{x}: H(x) \rightarrow H^{\prime}(x)$ is an $n$-equivalence for all $x \in X$. We would like to deduce that $\varphi_{A}: H(A) \rightarrow H^{\prime}(A)$ is an $n$-equivalence for all closed $A \subset X$. In case $H, H^{\prime}$ are nonnegative it follows from [7, Theorem 4.1] and Theorem (2.5). In [8, Corollary (4.7)] it was shown to follow if $X$ is a locally compact finite dimensional separable metric space. Below we show that the hypothesis of local compactness is unnecessary. First we prove a result about finite dimensional spaces. We use the definition of dimension denoted Ind in [5]. Thus, $X$ has dimension -1 if $X=\varnothing$, and for $m \geq 1$, $X$ has dimension $\leq m$ if every pair of disjoint closed subsets of $X$ can be separated by a closed set of dimension $\leq m-1$.

Lemma (5.3). Let $A, B$ be closed subsets of an m-dimensional space $X$ such that $A \cup B=\operatorname{int}_{A \cup B} A \cup$ int $_{A \cup B} B$. Then there exist closed sets $A^{\prime}$, $B^{\prime}$ of $X$ with $A^{\prime} \subset A, B^{\prime} \subset B, A \cup B=A^{\prime} \cup B^{\prime}$ and $\operatorname{dim} A^{\prime} \cap B^{\prime}<m$.

Proof. Let $C=A \cup B$-int ${ }_{A \cup B} B$ and $D=A \cup B$-int ${ }_{A \cup B} A$. Then $C$, $D$ are disjoint closed subsets of $A \cup B$. Since $\operatorname{dim}(A \cup B) \leq m$, there exists a closed subset $E \subset A \cup B$ with $\operatorname{dim} E<m$ which separates $C, D$. Therefore, $A \cup B-E=C^{\prime} \cup D^{\prime}$ where $C^{\prime}, D^{\prime}$ are each open in $A \cup B$ $-E$ (so open in $A \cup B$ ), $C^{\prime} \cap D^{\prime}=\varnothing$, and $C \subset C^{\prime}, D \subset D^{\prime}$. Let $A^{\prime}=C^{\prime} \cup E, B^{\prime}=D^{\prime} \cup E$. Then $A^{\prime}, B^{\prime}$ are closed subsets of $A \cup B$ (so closed in $X$ ), $A^{\prime} \subset A \cup B-D=\operatorname{int}_{A \cup B} A \subset A$, and similarly $B^{\prime} \subset B$, $A^{\prime} \cup B^{\prime}=A \cup B$ and

$$
\operatorname{dim} A^{\prime} \cap B^{\prime}=\operatorname{dim}\left[\left(C^{\prime} \cup E\right) \cap\left(D^{\prime} \cup E\right)\right]=\operatorname{dim} E<m .
$$

THEOREM (5.4). Let $\varphi: H, \delta \rightarrow H^{\prime}, \delta^{\prime}$ be a homomorphism between additive cohomology theories on a finite dimensional normal space $X$ both having paracompact supports and suppose there is $n \in \mathbf{Z}$ such that $\varphi_{x}$ : $H(x) \rightarrow H^{\prime}(x)$ is an n-equivalence for all $x \in X$. Then $\varphi_{A}: H(A) \rightarrow H^{\prime}(A)$ is an n-equivalence for all closed $A \subset X$. 
Proof. By Theorem (2.5) it suffices to prove the result in case $\mathrm{A}$ is a paracompact subset of $X$. We do this by induction on $\operatorname{dim} A$. If $\operatorname{dim} A=0$, $A=\varnothing$ and $\varphi_{A}$ is an isomorphism. Assume the result valid for all paracompact subsets of dimension $<m$ where $m \geq 1$ and let $A$ be a paracompact subset such that $\operatorname{dim} A=m$.

First, we show $\varphi_{A}: H^{q}(A) \rightarrow H^{q}(A)$ is an epimorphism for $q<n$. Let $u \in H^{\prime q}(A)$ be fixed and let $\mathscr{C}$ be the collection of all closed subsets $B \subset A$ such that $u \mid B \in \operatorname{im} \varphi_{B}$. The hypothesis on $\varphi_{x}$ and continuity of $H$, $H^{\prime}$ imply that every point of $A$ has a closed nbhd in $A$ which is an element of $\mathscr{C}$. From the definition of $\mathscr{C}$ it is clear that $B^{\prime} \subset B, B \in \mathscr{C}$ imply $B^{\prime} \in \mathscr{C}$. The additivity of $H, H^{\prime}$ imply that the union of a discrete family of elements of $\mathscr{C}$ is an element of $\mathscr{C}$.

We prove that $B, B^{\prime} \in \mathscr{C}$ and

$$
B \cup B^{\prime}=\operatorname{int}_{B \cup B^{\prime}} B \cup \operatorname{int}_{B \cup B^{\prime}} B^{\prime}
$$

imply $B \cup B^{\prime} \in \mathscr{C}$. By Lemma (5.3) there exist closed $C, C^{\prime}$ such that $C \subset B, C^{\prime} \subset B^{\prime}, B \cup B^{\prime}=C \cup C^{\prime}$ and $\operatorname{dim}\left(C \cap C^{\prime}\right)<m$. The following diagram has exact rows and commutes up to sign

$$
\begin{array}{ccccccc}
H^{q-1}\left(C \cap C^{\prime}\right) & \stackrel{\delta}{\rightarrow} & H^{q}\left(C \cup C^{\prime}\right) & \stackrel{\alpha}{\rightarrow} & H^{q}(C) \dot{\oplus} H^{q}\left(C^{\prime}\right) & \stackrel{\beta}{\rightarrow} & H^{q}\left(C \cap C^{\prime}\right) \\
\varphi \downarrow \approx & & \varphi \downarrow & & \downarrow \varphi & & \approx \downarrow \varphi \\
H^{\prime q-1}\left(C \cap C^{\prime}\right) & \stackrel{\delta^{\prime}}{\rightarrow} & H^{\prime q}\left(C \cup C^{\prime}\right) & \stackrel{\alpha^{\prime}}{\rightarrow} & H^{\prime q}(C) \oplus H^{\prime q}\left(C^{\prime}\right) & \stackrel{\beta^{\prime}}{\rightarrow} & H^{\prime q}\left(C \cap C^{\prime}\right)
\end{array}
$$

The two vertical maps on the ends are isomorphism because $\operatorname{dim}\left(C \cap C^{\prime}\right)$ $<m, q<n$, and the inductive hypothesis. It follows [7, part 2) of Lemma 2.19] that $\alpha^{\prime-1}(\operatorname{im} \varphi) \subset \operatorname{im} \varphi$. Since $C, C^{\prime} \in \mathscr{C},\left(u|C, u| C^{\prime}\right) \in \operatorname{im} \varphi$. Therefore, $u \mid\left(C \cup C^{\prime}\right) \in \operatorname{im} \varphi$. Since $C \cup C^{\prime}=B \cup B^{\prime}, B \cup B^{\prime} \in \mathscr{C}$. By [4, Theorem 5.5] $A \in \mathscr{C}$ so $u \in \operatorname{im} \varphi_{A}$.

Next, we show $\varphi_{A}: H^{q}(A) \rightarrow H^{\prime q}(A)$ is a monomorphism for $q \leq n$. Let $u \in \operatorname{ker} \varphi_{A}$ be fixed and let $\mathscr{C}$ be the collection of all closed subsets $B \subset A$ such that $u \mid B=0$. Again every point of $A$ has a closed nbhd in $A$ which is an element of $\mathscr{C}$, every closed subset of an element of $\mathscr{C}$ is an element of $\mathscr{C}$, and the union of a discrete family of elements of $\mathscr{C}$ is an element of $\mathscr{C}$. Using Lemma (5.5) as above, it is not hard to show that $B$, $B^{\prime} \in \mathscr{C}$ and

$$
B \cup B^{\prime}=\operatorname{int}_{B \cup B^{\prime}} B \cup \operatorname{int}_{B \cup B^{\prime}} B^{\prime}
$$

imply $B \cup B^{\prime} \in \mathscr{C}$. It follows again that $A \in \mathscr{C}$. 


\section{REFERENCES}

[1] G. Bredon, Sheaf Theory, Mc Graw-Hill, New York, N. Y. 1967.

[2] S. Eilenberg and N. E. Steenrod, Foundations of Algebraic Topology, Princeton University Press, Princeton, N. J., 1952.

[3] J. D. Lawson, Comparison of taut cohomologies, Aeq. Math., 2 (1973), 201-209.

[4] E. Michael, Local properties of topological spaces, Duke Math. J., 21 (1954), 163-171.

[5] K. Nagami, Dimension Theory, Academic Press, New York, N. Y. 1970.

[6] E. Spanier, Algebraic Topology, Springer Verlag, New York, N. Y. 1982.

[7] Cohomology isomorphisms, Contemporary Math., 12 (1982), 315-329.

[8] __ Cohomology theories on spaces, to appear.

Received October 22, 1984. The author gratefully acknowledges hospitality by the Institut des Hautes Etudes Scientifiques in Bures-sur-Yvette and partial support by the Max-Planck Institut für Mathematik in Bonn during the preparation of this year.

UNIVERSITY OF CALIFORNIA

BERKELEY, CA 94720 


\title{
PACIFIC JOURNAL OF MATHEMATICS EDITORS
}

\author{
V. S. VARADARAJAN \\ (Managing Editor) \\ University of California \\ Los Angeles, CA 90024 \\ HERBERT ClEMENS \\ University of Utah \\ Salt Lake City, UT 84112 \\ R. FINN \\ Stanford University \\ Stanford, CA 94305
}

\author{
HERMANN FLASCHKA \\ University of Arizona \\ Tucson, AZ 85721 \\ RAMESH A. GANGOLLI \\ University of Washington \\ Seattle, WA 98195 \\ VAUGHAN F. R. JONES \\ University of California \\ Berkeley, CA 94720 \\ ROBION KIRBY \\ University of California \\ Berkeley, CA 94720
}

C. C. MOORE

University of California

Berkeley, CA 94720

H. SAMELSON

Stanford University

Stanford, CA 94305

HAROLD STARK

University of California, San Diego

La Jolla, CA 92093

\section{ASSOCIATE EDITORS}

\author{
R. AREnS \\ E. F. BECKENBACH \\ B. H. NEUMANN \\ F. WOLF \\ K. YOSHIDA \\ (1906-1982)

\section{SUPPORTING INSTITUTIONS}

\begin{abstract}
UNIVERSITY OF ARIZONA
UNIVERSITY OF BRITISH COLUMBIA

UNIVERSITY OF CALIFORNIA

MONTANA STATE UNIVERSITY

UNIVERSITY OF NEVADA, RENO

NEW MEXICO STATE UNIVERSITY

OREGON STATE UNIVERSITY
\end{abstract} \\ CALIFORNIA INSTITUTE OF TECHNOLOGY \\ UNIVERSITY OF OREGON \\ UNIVERSITY OF SOUTHERN CALIFORNIA \\ STANFORD UNIVERSITY \\ UNIVERSITY OF HAWAII \\ UNIVERSITY OF TOKYO \\ UNIVERSITY OF UTAH \\ WASHINGTON STATE UNIVERSITY \\ UNIVERSITY OF WASHINGTON
}

The Supporting Institutions listed above contribute to the cost of publication of this Journal, but they are not owners or publishers and have no responsibility for its content or policies.

Mathematical papers intended for publication in the Pacific Journal of Mathematics should be in typed form or offset-reproduced (not dittoed), double spaced with large margins. Please do not use built up fractions in the text of the manuscript. However, you may use them in the displayed equations. Underline Greek letters in red, German in green, and script in blue. The first paragraph must be capable of being used separately as a synopsis of the entire paper. In particular it should contain no bibliographic references. Please propose a heading for the odd numbered pages of less than 35 characters. Manuscripts, in triplicate, may be sent to any one of the editors. Please classify according to the scheme of Math. Reviews, Index to Vol. 39. Supply name and address of author to whom proofs should be sent. All other communications should be addressed to the managing editor, or Elaine Barth, University of California, Los Angeles, California 90024.

There are page-charges associated with articles appearing in the Pacific Journal of Mathematics. These charges are expected to be paid by the author's University, Government Agency or Company. If the author or authors do not have access to such Institutional support these charges are waived. Single authors will receive 50 free reprints; joint authors will receive a total of 100 free reprints. Additional copies may be obtained at cost in multiples of 50 .

The Pacific Journal of Mathematics is issued monthly as of January 1966. Regular subscription rate: $\$ 190.00$ a year (5 Vols., 10 issues). Special rate: $\$ 95.00$ a year to individual members of supporting institutions.

Subscriptions, orders for numbers issued in the last three calendar years, and changes of address should be sent to Pacific Journal of Mathematics, P.O. Box 969, Carmel Valley, CA 93924, U.S.A. Old back numbers obtainable from Kraus Periodicals Co., Route 100, Millwood, NY 10546.

The Pacific Journal of Mathematics at P.O. Box 969, Carmel Valley, CA 93924 (ISSN 0030-8730) publishes 5 volumes per year. Application to mail at Second-class postage rates is pending at Carmel Valley, California, and additional mailing offices. Postmaster: send address changes to Pacific Journal of Mathematics, P.O. Box 969, Carmel Valley, CA 93924.

PUBLISHED BY PACIFIC JOURNAL OF MATHEMATICS, A NON-PROFIT CORPORATION

Copyright (C) 1986 by Pacific Journal of Mathematics 


\section{Pacific Journal of Mathematics}

\section{Vol. 123, No. $2 \quad$ April, 1986}

David Jay Anick, A loop space whose homology has torsion of all orders . . 257 Steven P. Diaz, Space curves that intersect often ................. 263

Thierry Fack and Hideki Kosaki, Generalized $s$-numbers of $\tau$-measurable

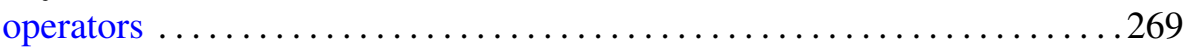

Karl Heinrich Hofmann and Karl Strambach, Lie's fundamental

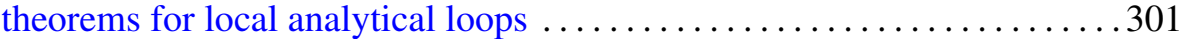

James Secord Howland, On the Kato-Rosenblum theorem ............. 329

Frieder Knüppel and Edzard Salow, Plane elliptic geometry over rings . . . . 337

Alan Noell, Peak points in boundaries not of finite type ................ 385

William J. Ralph, An extension of singular homology to Banach

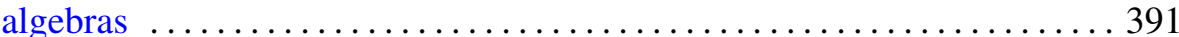

Wade C. Ramey, Averaging properties of pluriharmonic boundary values . . 407

Thomas Joseph Ransford, On the range of an analytic multivalued

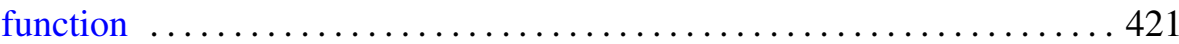

Christopher Donald Sogge, On restriction theorems of maximal-type . . . . 441

Edwin Spanier, Cohomology with supports $\ldots \ldots \ldots \ldots \ldots \ldots \ldots \ldots \ldots 47$

Emil J. Straube, Orthogonal projections onto subspaces of the harmonic

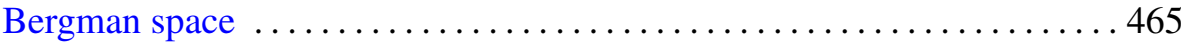

Thomas Vogel, Asymptotic behavior of two semilinear elliptic free boundary problems $\ldots \ldots \ldots \ldots \ldots \ldots \ldots \ldots \ldots$ 